Rev Inv Vet Perú 2014; 25(2): 317-323

doi: http://doi.org/10.15381/rivep.v25i2.8504

\title{
Comunicación
}

\section{INFECCIÓN POR Klebsiella pneumoniae EN UN MONO CHORO (Lagothrix lagotricha) CRIADO COMO MASCOTA EN LIMA, PERÚ}

\author{
KLebsiella PNEUmoniae Infection in a Woolly Monkey (LaGothriX \\ lagotricha) Kept as Pet in Lima, Peru
}

\author{
Miryam Quevedo U., ${ }^{1,2}$ Jesús Lescano G. ${ }^{1}$
}

\section{RESUMEN}

Se reporta el caso de un mono choro (Lagothrix lagotricha) criado como mascota que llega a la consulta por presentar una tumefacción en el cuello, decaimiento e inapetencia. La tumefacción correspondió a un absceso. El examen radiográfico indicó signos radiográficos de bronconeumonía, la tinción alcohol-ácido descartó la presencia de micobacterias y en el cultivo bacteriológico se aisló Klebsiella pneumoniae. Se administró tratamiento antibiótico a base de enrofloxacina $5 \mathrm{mg} / \mathrm{kg}$ i.m. c/24 h durante 10 días, antibiótico susceptible según el antibiograma. El individuo se recuperó.

Palabras clave: primates, enfermedad, absceso, zoonosis

\section{Abstract}

A captive woolly monkey (Lagothrix lagotricha) maintained as pet was brought to the clinic due to the presence of a swelling in the neck, loss of appetite and weakness. The swelling corresponded to an abscess. The radiographic test showed signs of bronchopneumonia, the acid alcohol test ruled out the presence of mycobacteria and in the bacterial culture Klebsiella pneumoniae was isolated. Antibiotic therapy, based on enrofloxacin $5 \mathrm{mg} / \mathrm{kg}$ i.m. daily was administered for 10 days, as this antibiotic showed high susceptibility in the antibiogram. The patient fully recovered.

Key words: primates, disease, abscess, zoonoses

\footnotetext{
${ }^{1}$ Laboratorio de Anatomía Animal y Fauna Silvestre, Facultad de Medicina Veterinaria, Universidad Nacional Mayor de San Marcos, Lima

${ }^{2}$ E-mail: mquevedo@veterinaria-unmsm.edu.pe
}

Recibido: 3 de junio de 2013

Aceptado para publicación: 20 de enero de 2014 


\section{INTRODUCCIÓN}

La tenencia de animales silvestres como mascotas ha sido una costumbre a lo largo de la historia de la humanidad, la cual prevalece hasta hoy en día, tanto en sociedades rurales como urbanas (Drews, 1999). Sin embargo, dicha actividad es una amenaza para la salud pública, ya que se incrementa el riesgo de exposición a agentes zoonóticos. Se estima que las zoonosis representan el $75 \%$ de las infecciones emergentes, cuyo principal y usualmente desconocido reservorio lo conforman los animales silvestres (principalmente mamíferos), quienes además pueden ser fuente de reemergencia de enfermedades infecciosas previamente controladas (Perkins et al., 2005; Kuiken et al., 2005; Chomel et al., 2007, Monsalve et al., 2009; Rhyan y Spraker, 2010).

La literatura reporta diversos ejemplos de brotes de enfermedades zoonóticas asociados a la tenencia de animales silvestres como mascotas. Uno de estos casos ocurrió en Ceará, Brasil, donde se presentó un brote de rabia asociado a una nueva variante viral que provino de un mono tití (Callithrix jacchus jacchus) mantenido como mascota (Chomel et al., 2007). También puede ocurrir el pasaje de agentes infecciosos desde las personas hacia la fauna silvestre (antropozoonosis), como en los casos de mortalidad de monos titíes (Callithrix spp) asociada a infección por herpesvirus humano tipo 1, que fueron reportados en Brasil y Austria (Bruno et al., 1997; Huemer et al., 2002; Longa et al., 2011).

Una de las enfermedades infecciosas emergentes en humanos cuya epidemiología viene aparentemente cambiando es causada por Klebsiella pneumoniae. Esta es una bacteria aeróbica gram negativa, causante de una variedad de infecciones que incluyen casos fatales acompañados de lesiones entre las que se encuentran abscesos hepáticos y cerebrales, émbolos o abscesos pulmonares, endoftalmitis, meningitis y osteomielitis. Las cepas invasivas de $K$. pneumoniae causantes de mortalidad en el humano han sido asociadas a la presencia del fenotipo de hipermucoviscosidad y, además, manifiestan los serotipos capsulares K1 y K2 (Twenhafel et al., 2008; Jang et al., 2010; Whitehouse et al., 2010).

$K$. pneumoniae ha sido reportado en especies de primates no humanos del Viejo Mundo (Chlorocebus aethiops) y Neotropicales (Aotus spp), hallándosele tanto en la flora oral y fecal (sin producir enfermedad clínica), como en individuos enfermos (produciendo neumonía, meningitis, peritonitis, cistitis, abscesos multisistémicos y septicemia). Además, se ha encontrado evidencia serológica de exposición a $K$. pneumoniae en mamíferos silvestres de vida libre (Zalophus californianus) y mantenidos en cautiverio (Aotus lemurinus). También se ha reportado mortalidad asociada a infección por este agente en fauna silvestre cautiva y libre (Obaldia, 1991; Sánchez et al., 2006; Twenhafel et al., 2008; Jang et al., 2010; Whitehouse et al., 2010; Liao et al., 2011).

\section{Caso Clínico}

\section{Historia Clínica}

Llega a la Clínica de Animales Silvestres y Exóticos de la Facultad de Medicina Veterinaria (FMV), Universidad Nacional Mayor de San Marcos (UNMSM), Lima, un mono choro (Lagothrix lagotricha) macho de 3.5 años de edad, el cual ha sido criado en cautiverio durante tres años. Durante este periodo, el individuo había sido alimentado con una dieta compuesta principalmente de frutas y ocasionalmente con vegetales. De acuerdo al propietario, el animal presentó dos días antes una tumefacción en el cuello. Además, presentó fiebre, anorexia y decaimiento, siendo medicado empíricamente con acetaminofén y amoxicilina. El motivo de la consulta fue el aumento de tamaño de la tumefacción y la falta de respuesta al tratamiento. 


\section{Examen Clínico}

El animal presenta buena condición corporal y moderada depresión. Presenta una tumefacción de 8 x $5 \mathrm{~cm}$ en la región cervical ventral derecha, de consistencia firme, superficie lisa, y sin movilidad a la palpación.

Debido al estado depresivo del animal, se postergó la toma de muestras y exámenes complementarios hasta su estabilización, iniciándose el tratamiento con enrofloxacina $5 \mathrm{mg} / \mathrm{kg}$ i.m. c/24 h, ketoprofeno $5 \mathrm{mg} / \mathrm{kg}$ i.m. c/24 h (Carpenter, 2005) y fluidoterapia vía oral. El animal presentó mejor ánimo e hiporexia al tercer día del tratamiento, aunque los dueños manifestaron que tuvo fiebre los dos días anteriores, y que fuera controlada con acetaminofén $5 \mathrm{mg} / \mathrm{kg} \mathrm{VO}$, por indicación médica veterinaria.

Desde el primer momento se recomendó a los propietarios minimizar el contacto e interacción con el animal debido a la posibilidad de que la enfermedad fuera causada por un agente zoonótico.

\section{Exámenes Complementarios}

Una vez estabilizado el animal, se procedió a realizar exámenes complementarios. El animal fue contenido químicamente para la colección de muestras y toma de placas radiográficas con una asociación de clorhidrato de ketamina $3 \mathrm{mg} / \mathrm{kg}$ y clorhidrato de dexmedetomidina $25 \mu \mathrm{g} / \mathrm{kg}$ i.m. Se observó un tiempo de inducción de 6 minutos, un tiempo de sedación de 20 minutos y un tiempo de recuperación de 2 minutos. La reversión del clorhidrato de dexmedetomidina se hizo con clorhidrato de atipamezol $125 \mu \mathrm{g} / \mathrm{kg}$ i.m. El procedimiento se realizó sin complicación alguna.

Se tomó un aspirado de la tumefacción mediante punción con aguja fina (21 G x 1") y sangre entera (con EDTA) mediante punción de la vena femoral derecha. Con la muestra de aspirado se hizo un examen citológico, tinción alcohol-ácido resistente, cultivo bacteriológico y antibiograma, y con la sangre se hizo un examen hematológico completo en los laboratorios de la FMV. Además, se tomaron radiografías torácicas (vistas latero-lateral izquierda y ventro-dorsal) en la Clínica de Animales Menores de la FMV. Los resultados de los exámenes realizados se presentan en el Cuadro 1.

\section{Diagnóstico y Tratamiento}

El diagnóstico fue de absceso subcutáneo asociado a Klebsiella pneumoniae. En base a los resultados, se continuó el tratamiento con enrofloxacina $5 \mathrm{mg} / \mathrm{kg}$ i.m. c/24 h hasta completar 10 días de terapia. $\mathrm{Al}$ décimo día el paciente se encontraba activo y se alimentaba adecuadamente. La tumefacción había disminuido de tamaño ( 2 x $1 \mathrm{~cm}$ aprox.). Asimismo, el propietario manifiesta haber observado el drenaje de un líquido amarillento verdoso a través del punto de incisión realizado para la toma de muestras. Se suspende la antibioticoterapia y se le vuelve a evaluar cuatro días más tarde, encontrándosele totalmente recuperado.

\section{Discusión}

En base a la anamnesis y examen físico realizados al paciente, se postularon dos diagnósticos diferenciales: un proceso neoplásico (pudiendo ser carcinoma de células escamosas o linfosarcoma) (Ritcher y Buyukmihci, 1979; Kagira et al., 2007) o un proceso infeccioso (como un absceso subcutáneo o tuberculosis nodular) (Richard, 1989; Thoen et al., 2009).

Los resultados permitieron demostrar que la tumefacción en el cuello del paciente correspondía a un proceso inflamatorio infeccioso supurativo asociado a la presencia de la bacteria $K$. pneumoniae, la cual era susceptible a enrofloxacina, ciprofloxacina y gentamicina. 
Cuadro 1. Resultados de los exámenes de patología clínica, bacteriología e imagenología en un mono choro (Lagothrix lagotricha) con absceso subcutáneo asociado a Klebsiella pneumoniae

Patología clínica

Examen citológico

Hematología

Eritrocitos $\left(\mathrm{x} 10^{6} / \mu \mathrm{l}\right)$

Hemoglobina $(\mathrm{g} / \mathrm{dl})$

Hematocrito (\%)

$\operatorname{VCM}(\mathrm{fl})$

HCM (pg)

$\mathrm{CHCM}(\mathrm{g} / \mathrm{dl})$

Plaquetas $(\mathrm{x} \mu \mathrm{l})$

Leucocitos $\left(\mathrm{x} 10^{3} / \mu \mathrm{l}\right)$

Neutrófilos

Mielocitos

Metacariocitos

Abastonados

Segmentados

Linfocitos (\%)

Monocitos (\%)

Eosinófilos (\%)

Basófilos (\%)

Anisocitosis

Pilas globulares

Macroplaquetas

Células atípicas

Examen bacteriológico

Tinción alcohol-ácid o resistente

Cultivo bacteriológico

Antibiograma

Imagenología

Diagnóstico radiográfico
Se observan abundantes neutrófilos intactos y degenerados. Escasos macrófagos

$\begin{array}{cc} & \text { Valor referencial }^{1} \\ 3.1 & 2.8-7.15 \\ 8.7 & 10-15 \\ 28 & 29-49 \\ 32 & 59.5-104.3 \\ 28 & 19.6-38.3 \\ 21 & 31.8-47.2 \\ 530000 & 100000-347000 \\ 16100 & 4700-29400\end{array}$

Valor relativo (\%)
0
0
0
70
30
0
0
0

Valor absoluto $(x \mu l)$

0
0
0
11270
4830
0
0
0

$+$

$++$

$+$

No se observan

Negativo

Klebsiella pneumoniae

Sensible a enrofloxacina, ciprofloxacina y gentamicina. Resistente a amoxicilina

Signos radiográficos de bronconeumonía en lóbulos pulmonares caudales

${ }^{1}$ Valores referenciales obtenidos del Internacional Species Information System 1999.

La tinción alcohol ácido resistente permitió descartar la presencia de micobacterias $\mathrm{y}$ un posible caso de tuberculosis nodular. Por su parte, el examen radiológico indicó signos compatibles con bronconeumonía en los lóbulos caudales, la cual no se manifestó clínicamente (ausencia de estertores y secreción nasal) durante la evaluación del animal. $\mathrm{Al}$ respecto, cabe señalar que actualmente la historia clínica y el examen físico no se consideran herramientas sensibles para el diagnóstico de neumonía en pacientes humanos, 
mientras que el examen radiológico es considerado el mejor método diagnóstico para dicha patología (Gil et al., 2005; Moénne, 2013). El hallazgo radiológico podría también estar asociado a $K$. pneumoniae ya que han sido reportados casos causados por este patógeno (Sánchez et al., 2006).

El hemograma indica anemia microcítica hipocrómica leve al comparar los valores encontrados con los reportados por ISIS (1999). La anemia estuvo posiblemente asociada a deficiencia de hierro producto de una dieta deficitaria de este mineral. Es conocido que la dieta de L. lagotricha mantenidos en cautiverio debe estar compuesta de hojas, frutas y flores (Rylands et al., 2001), e incluso se reporta el consumo de artrópodos (Stevenson et al., 1994); sin embargo, la dieta que recibió el paciente no fue diseñada considerando los requerimientos nutricionales de la especie ni el aporte nutricional de cada ítem alimenticio, por el contrario, estaba supeditada a la disponibilidad de insumos caseros.

La trombocitosis y presencia de macroplaquetas indica una mayor demanda de trombocitos, lo cual estaría asociado a la presencia de microhemorragias, producto del proceso inflamatorio manifiesto a través del hallazgo de pilas globulares. Sin embargo, el leucograma no presenta alteraciones, por lo que se asume que el cuadro infeccioso era leve y localizado. Cabe señalar que en procesos neumónicos en pacientes humanos se puede hallar trombocitosis, anemia y ausencia de alteraciones en el leucograma (Mantadakis et al., 2008; Reade et al., 2010, Andrés Martín et al., 2012), siendo posible que estos hallazgos se observen en primates no humanos que padecen dicha patología.

\section{Literatura Citada}

1. Andrés Martín A, Moreno-Pérez, D, Alfayate Miguélez S, Couceiro Gianzo JA, García García ML, Korta Murua J, Martínez León MI, et al. 2012. Etiología y diagnóstico de la neumonía ad- quirida en la comunidad y sus formas complicadas. An Pediatr (Barc) 76(3): 162.e1-162.e18

2. Bruno SF, Liebhold MM, MätzRensing K, Romao MA, Didier A, Brandes $F$, et al. 1997. Herpesvirus infections in free living black tufted ear marmosets (Callithrix penicillata, E. Geoffroyi 1812) at the State Park of Serra da Tiririca, Niterói, Rio de Janeiro, Brazil. Berl Munch Tierarztl Wochenschr 110:427-430.

3. Carpenter JW. 2005. Exotic animal formulary. $3^{\text {rd }}$ ed. USA: Elsevier Saunders. p 495-534.

4. Chomel BB, Belotto A, Meslin FX. 2007. Wildlife, exotic pets, and emerging zoonoses. Emerg Infect Dis 13: 6-11.

5. Drews C. 1999. Simpatía y empatía hacia la fauna: raíces de la tenencia de mascotas silvestres. En: Drews C (ed). Rescate de fauna en el neotrópico. Heredia, Costa Rica: Universidad Nacional EUNA. p 31-52.

6. Gil R, Fernández P, Sabbagh E. 2005. Diagnóstico clínico-radiológico de la neumonía del adulto adquirida en la comunidad. Rev Chil Enf Respir 21: 89-94.

7. Huemer HP, Larcher C, CzedikEysenberg T, Nowotny N, Reifinger $M$. 2002. Fatal infection of a pet monkey with human herpesvirus 1. Emerg Infect Dis 8: 639-641.

8. [ISIS] International species information system. 1999. Physiological data reference values - Woolly monkey Lagothrix lagotricha. Apple Valley, Minnesota, USA. [Internet]. Disponible en: www.isis.org

9. Jang $S$, Wheeler L, Carey RB, Jensen $B$, Crandall CM, Schrader KN, Jessup D, et al. 2010. Pleuritis and suppurative pneumonia associated with a hypermucoviscosity phenotype of Klebsiella pneumoniae in California sea lions (Zalophus californianus). Vet Microbiol 141: 174-177.

10. Kagira JM, Ngotho M, Thuitu JK, Jensen HE, Hau J. 2007. Lymphosarcoma in adult African green 
monkeys (Chlorocebus aethiops). Scand J Lab Anim Sci 34: 241-247.

11. Kuiken T, Leighton FA, Fouchier RA, LeDuc JW, Peiris JS, Schudel A, et al. 2005. Public health: pathogen surveillance in animals. Science 309: 1680-1681.

12. Liao CH, Huang YT, Lai CC, Chang CY, Chu FY, Hsu MS, et al. 2011. Klebsiella pneumoniae bacteremia and capsular serotypes, Taiwan. Emerg Infect Dis 17: 113-1115.

13. Longa CS, Bruno SF, Pires AR, Romijn PC, Kimura LS, Costa CHC. 2011. Human herpesvirus 1 in wild marmosets, Brazil, 2008. Emerg Infect Dis 17: 1308-1309.

14. Matadankis E, Tsalkidis A, Chatzimichael A. 2008. Thrombocytosis in childhood. Indian Pediatr 45: 669677.

15. Moénne K. 2013. Neumonías adquiridas en la comunidad en niños: diagnóstico por imágenes. Rev Med Clín Condes 24(1):27-35.

16. Monsalve S, Mattar S, Gonzalez M. 2009. Zoonosis transmitidas por animales silvestres y su impacto en las enfermedades emergentes y reemergentes. Rev MVZ Córdoba 14(2): 1762-1773.

17. Obaldía N. 1991. Detection of Klebsiella pneumoniae antibodies in Aotus l. lemurinus (Panamanian owl monkey) using an enzyme linked immunosorbent assay (ELISA) test. Lab Anim 25: 133-141.

18. Perkins SE, Cattadori I, Hudson PJ. 2005. The role of mammals in emerging zoonoses. Mammal Study 30: S67-S71.

19. Reade MC, Weissfeld L, Angus DC, Kellum JA, Milbrandt EB. 2010. The prevalence of anemia and its association with 90-day mortality in hospitalized community-acquired pneumonia. BMC Pulm Med 10: 15.

20. Richard C. 1989. Epidemiology of Klebsiella pneumoniae infections in 2 colonies of squirrel monkeys and lemurs. Bull Soc Pathol Exot Filiales 82: 458-464.

21. Rhyan JC, Spraker TR. 2010. Emergence of diseases from wildlife reservoirs. Vet Pathol 47: 34-39.

22. Ritcher CB, Buykmihci N. 1979. Squamous cell carcinoma of the epidermis in an aged white-lipped tamarin (Saguinus Fuscicollis Leucogenys Gray). Vet Pathol 16: 263-265.

23. Rylands $A B$, Valladares-Pádua $C$, da Rocha e Silva R, Boere V, Catáo-Dias $J L$, Passinatti A, de Barros Vaz Guimaráes MA. 2001. Order Primates. En: Fowler ME, Cubas ZS (eds). Biology, medicine, and surgery of South American wild animals. USA: Iowa University Press. p 256-278.

24. Sánchez N, Gálvez H, Montoya E, Gozalo A. 2006. Mortalidad en crías de Aotus sp (Primates: Cebidae) en cautiverio: una limitante para estudios biomédicos con modelos animales. Rev Peru Med Exp Salud Públ 23: 221-224.

25. Stevenson PR, Quiñones MJ, Ahumana JA. 1994. Ecological strategies of woolly monkeys (Lagothrix lagotricha) at Tinigua National Park, Colombia. Am J Primatol 32: 123-140.

26. Thoen CO, Lobue PA, Enarson DA, Kaneene JB, de Kantor IN. 2009. Tuberculosis: a re-emerging disease in animals and humans. Vet Ital 45: 135-181.

27. Twenhafel NA, Whitehouse CA, Stevens EL, Hottel HE, Foster CD, Gamble S, et al. 2008. Multisystemic abscesses in African green monkeys (Chlorocebus aethiops) with invasive Klebsiella pneumoniae - Identification of the hypermucoviscosity phenotype. Vet Pathol 45: 226-231.

28. Vaughan TA, Ryan JM, Czaplewski NJ. 2010. Mammalian disease and zoonoses. En: Mammalogy. $5^{\text {th }}$ ed. USA: Jones and Bartlett Publ. p W-19.

29. West G, Heard D, Caulkett N. 2007. Zoo animal and wildlife immobilization and anaesthesia. USA: Blackwell Publ. 718 p. 
30. Whitehouse CA, Keirstead N, Taylor $J$, Reinhardt JL, Beierschmitt A. 2010. Prevalence or hypermucoid Klebsiella pneumoniae among wild- caught and captive vervet monkeys (Chlorocebus aethiops sebaeus) on the Island of St. Kitts. J Wildlife Dis 46: 971976. 